\title{
Acute versus long-term effects of 6-hydroxydopamine on oxidative stress and dopamine depletion in the striatum of mice
}

\author{
Mustafa Varcin ${ }^{\mathrm{a}}$, Eduard Bentea ${ }^{\mathrm{a}}$, Birgit Mertens ${ }^{\mathrm{a}}$, Chris Van Den Haute ${ }^{\mathrm{b}}$, Veerle Baekelandt ${ }^{\mathrm{b}}$, \\ Yvette Michotte ${ }^{a}$, Sophie Sarre ${ }^{\mathrm{a}, *}$ \\ a Department of Pharmaceutical Chemistry and Drug Analysis, Center for Neuroscience, Vrije Universiteit Brussel, Laarbeeklaan 103, B-1090 Brussels, Belgium \\ ${ }^{\mathrm{b}}$ Laboratory for Neurobiology and Gene Therapy, Katholieke Universiteit Leuven, Belgium
}

\section{A R T I C L E I N F O}

\section{Article history:}

Received 26 January 2011

Received in revised form 4 July 2011

Accepted 7 July 2011

\section{Keywords:}

6-Hydroxydopamine

Mice

Striatum

Oxidative stress

Microdialysis

Salicylate trapping

\begin{abstract}
A B S T R A C T
Oxidative stress is one of the mechanisms which may be important in the pathogenesis of Parkinson's disease. In the current study, the effects of 6-hydroxydopamine (6-OHDA) perfusion on hydroxyl radical formation in the mouse striatum were investigated using the in vivo salicylate trapping microdialysis technique. The latter uses salicylate as a trapping agent for hydroxyl radicals with formation of 2,3-dihydroxybenzoic acid (2,3-DHBA), which is measured by HPLC. Two different approaches of the technique were validated in mice. First, perfusion of the trapping agent salicylate $(1 \mathrm{mM})$ via the probe in combination with 6-OHDA $(5 \mu \mathrm{M})$ was used to screen for radical scavenging properties of compounds in mice. Alternatively, striatal administration of 6-OHDA in a concentration known to induce nigrostriatal denervation $(1 \mathrm{mM})$, without the trapping agent, allowed to maximally challenge the neuronal microenvironment and as such to investigate both its acute and long-term effects. In the first method, as expected, glutathione (GSH) $(1.5 \mathrm{mM})$ prevented the 6-OHDA-induced increase in 2,3-DHBA levels. In the second method, GSH prevented the hydroxyl radical formation, while depletion of GSH with 2-cyclohexen-1one ( $\mathrm{CHO}$ ) resulted in significantly higher 2,3-DHBA levels than when 6-OHDA was perfused alone. Three weeks after the local 6-OHDA perfusion, the total striatal dopamine (DA) and dihydroxyphenylacetic acid (DOPAC) content were reduced by $30 \%$, compared to the intact striatum, accompanied by a reduction in striatal tyrosine hydroxylase $(\mathrm{TH})$ immunoreactive (ir) nerve terminals. This suggests that the second method can be used to determine the acute as well as the long-term effects of 6-OHDA in the mouse striatum.
\end{abstract}

(C) 2011 Elsevier B.V. All rights reserved.

\section{Introduction}

Several studies suggest that Parkinson's disease is a multifactorial disorder. Even though the precise etiology of Parkinson's disease remains uncertain, genetic and/or environmental factors are clearly important in its pathogenesis (Aoyama et al., 2008; Meredith et al., 2008; Obeso et al., 2010; Przedborski, 2005) among which oxidative stress (Koutsilieri et al., 2002; Yacoubian and Standaert, 2009) is one of the most commonly accepted mechanisms (Aoyama et al., 2008; Garcia et al., 2000; Jenner, 2003; Schulz et al., 2000). Oxidative stress can be described as a condition in

Abbreviations: 2,3-DHBA, 2,3-dihydroxybenzoic acid; 2,5-DHBA, 2,5dihydroxybenzoic acid; 6-OHDA, 6-hydroxydopamine; CHO, 2-cyclohexene-1-one; DA, dopamine; DOPAC, 3,4-dihydroxyphenylacetic acid; GSH, glutathione; LC, liquid chromatography; MPTP, 1-methyl-4-phenyl-1,2,3,6-tetrahydropyridine; PEEK, polyetheretherketone; ROS, reactive oxygen species; $\mathrm{TH}$, tyrosine hydroxylase; ir, immunoreactive.

* Corresponding author. Tel.: +32 247747 46; fax: +32 24774113.

E-mail address: Sophie.Sarre@vub.ac.be (S. Sarre). which the cellular antioxidant defense mechanisms are insufficient to keep the level of reactive oxygen species (ROS) below a toxic threshold (Schulz et al., 2000). This may be either due to an overproduction of reactive free radicals or to a failure of cell buffering mechanisms (Yacoubian and Standaert, 2009). The overabundance of free radicals may damage cell lipids, proteins and DNA (Shimizu et al., 2002). Several of the genes linked to familial forms of Parkinson's disease appear to be involved in the protection against or the propagation of oxidative stress (Toulouse and Sullivan, 2008; Yacoubian and Standaert, 2009).

Among the ROS, the hydroxyl radical is the most reactive and destructive free radical. Due to its short lifetime and high reactivity, the direct measurement of hydroxyl radical formation in biological systems is difficult (Themann et al., 2001). Several analytical approaches are described to indirectly monitor the hydroxyl radical generation in vivo, by measuring the basal hydroxyl radical level, the evoked hydroxyl radical level, or both (Cheng et al., 2002; Di Giovanni et al., 2009). Among these techniques, in vivo salicylate trapping microdialysis is one of the most widely used to indirectly quantify the hydroxyl radicals in brain structures. In this 


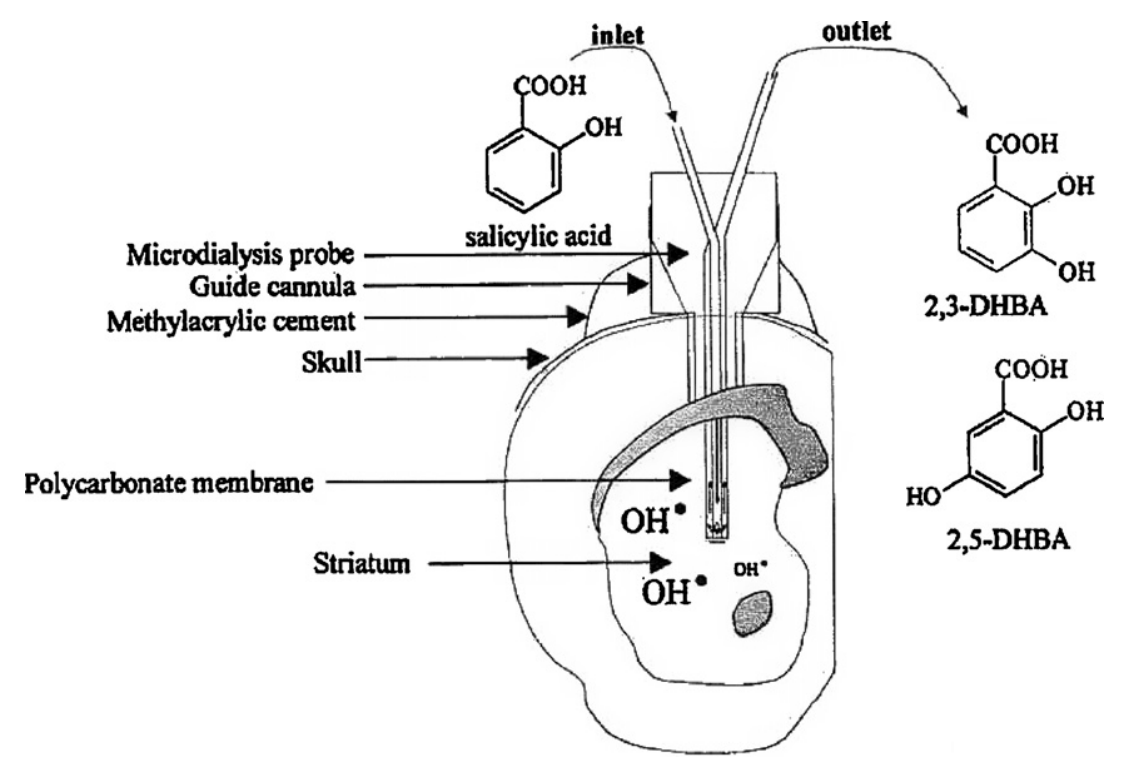

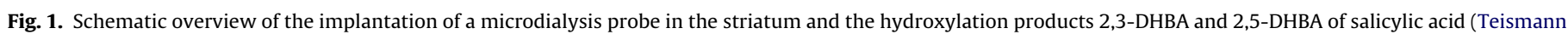
and Ferger, 2000). 2,3-DHBA: 2,3-dihydroxybenzoic acid; 2,5-DHBA: 2,5-dihydroxybenzoic acid; •OH: hydroxyl radical.

indirect method, salicylate, used as a trapping agent in the perfusate, reacts with hydroxyl radicals and based on the principle of aromatic hydroxylation forms 2,3-dihydroxybenzoic acid (2,3DHBA) and 2,5-dihydroxybenzoic acid (2,5-DHBA) (Fig. 1). Only the 2,3-DHBA content is quantified, as 2,5-DHBA can be formed endogenously, while all 2,3-DHBA is derived from the reaction (Di Giovanni et al., 2009; Teismann and Ferger, 2000). The 2,3-DHBA content in the dialysates is measured by HPLC with electrochemical detection.

Usually, in vivo salicylate trapping microdialysis is used as a screening method for possible therapeutic radical scavenging properties of specific compounds (Ferger et al., 2000; Obata, 2002; Opacka-Juffry et al., 1998; Yuan et al., 2004). In this approach, radical-enhancing molecules such as 1-methyl4-phenyl-2,3-dihydropyridinium ion or 6-OHDA are perfused to induce hydroxyl radical formation. Radical scavengers are able to block this formation (Cheng et al., 2002; Di Giovanni et al., 2009; Gruber et al., 2006; Teismann and Ferger, 2000). However, the method with 6-OHDA has not been applied in mice before.

The in vivo salicylate trapping technique may also be interesting for the study of acute oxidative stress and its long term consequences by determination of hydroxyl radical formation after 6-OHDA perfusion. However, exploration of the susceptibility of striatal dopaminergic nerve terminals for neurotoxins such as 6OHDA may be hampered in the traditional set-up since combined perfusion of 6-OHDA and the trapping agent via the microdialysis probe in the brain (Di Giovanni et al., 2009) may not be challenging enough for the neuronal microenvironment, due to the fact that the hydroxyl radicals are quickly captured by the trapping agent.

In this study, the in vivo salicylate trapping microdialysis technique as described by us (Yuan et al., 2004) was miniaturized for application in the mouse striatum in order to screen the possible radical scavenging properties of potential neuroprotectants. 6-OHDA was used to induce hydroxyl radical formation, and GSH as radical scavenger (Soto-Otero et al., 2000).

Furthermore, the method was slightly modified to allow the screening for differences in acute oxidative stress in vivo. In this new approach, 6-OHDA was perfused in the same concentration used to generate a striatal 6-OHDA lesion in mice. By doing so, insights into the acute, primary as well as long-term effects of the 6-OHDA neurotoxicity in the mouse striatum can be obtained.
In order to establish whether the modified method allows us to determine differences in susceptibility to oxidative stress, besides the use of GSH, mouse brain glutathione was depleted by 2-cyclohexene-1-one ( $\mathrm{CHO}$ ). $\mathrm{CHO}$ is an electrophilic agent that reduces GSH levels by conjugation through the GSH S-transferases (Choy et al., 2010; Masukawa et al., 1989; Pileblad and Magnusson, 1990). Depletion of GSH should enhance 6-OHDA-induced generation of oxidative stress.

By modulating the levels of endogenous GSH, either by supplementation, or by depletion, we show that our method is sensitive to variations in endogenous antioxidant mechanisms, and as a consequence can detect changes in 6-OHDA-induced hydroxyl radical production in different in vivo situations.

\section{Materials and methods}

All the chemical compounds where no supplier is mentioned, are supplied by Sigma-Aldrich, Brussels, Belgium.

\subsection{Animals}

In all experiments, C57BL/6J female mice (Charles River, France) of about 3 months old and weighing 20-25 g were used. Animals were kept under standardized conditions $\left(25^{\circ} \mathrm{C}, 12 \mathrm{~h}\right.$ light-darkcycle) with free access to food and tap water. At the end of the experiments, mice were killed with an overdose of pentobarbital (Nembutal ${ }^{\circledR}$, Ceva Sante Animale, Brussels, Belgium). Animal experiments were carried out according to the national guidelines on animal experimentation and were approved by the Ethical Committee for Animal Experiments of the Faculty of Medicine and Pharmacy of the Vrije Universiteit Brussel. All efforts were made to minimize animal suffering and the minimal number of animals necessary to produce reliable scientific data was used.

\subsection{Microdialysis: measurement of extracellular hydroxyl radicals}

\subsubsection{Stereotactic implantation of the microdialysis probe}

Mice were first anaesthetized with a mixture of ketamine $\left(100 \mathrm{mg} / \mathrm{kg}\right.$ i.p.; Ketamine $1000 \mathrm{Ceva}^{\circledR}$, Ceva Sante Animale, Brussels, Belgium) and xylazine ( $10 \mathrm{mg} / \mathrm{kg}$ i.p.; Rompun ${ }^{\circledR} 2 \%$, Bayer N.V., 
Brussels, Belgium) and placed on a Kopf stereotaxic frame. After positioning, an incision was made in the scalp and a section of the skull was exposed. The fascia and pericranial connective tissue were removed and a small hole was drilled through the dura mater and the skull. Next, the microdialysis guide (CMA/7 guide cannulas, CMA Microdialysis AB, Solna, Sweden) was implanted into the left striatum at the following coordinates relative to bregma L: -1.8 , A: +0.6 and V: +2.0 , according to the atlas of Franklin and Paxinos (Franklin and Paxinos, 1997). The guide was fixed to the skull with dental cement (Durelon ${ }^{\circledR}$ Carboxylate cement powder, 3M Espe AG, Seefeld, Germany). After the surgery, a probe (CMA/7, CMA Microdialysis AB, Solna, Sweden) with a membrane of $2 \mathrm{~mm}$ length and 6000 Da molecular weight cut-off was introduced via the cannula.

After probe insertion, mice were fitted with a plastic collar, placed in individual microdialysis cages allowing unrestricted movement (Raturn Cage System, BASi, USA) and allowed to recover overnight while they were perfused with modified Ringer's solution ( $147 \mathrm{mM} \mathrm{NaCl}, 4 \mathrm{mM} \mathrm{KCl}, 1.1 \mathrm{mM} \mathrm{CaCl} 2 \cdot 6 \mathrm{H}_{2} \mathrm{O}$ ). The perfusate was delivered at a constant flow rate of $2 \mu \mathrm{l} / \mathrm{min}$ using $2.5 \mathrm{ml}$ calibrated microsyringe gastight (Hamilton CO, Reno, USA) attached to a CMA/100 Microdialysis Pump (CMA Microdialysis AB, Solna, Sweden). Approximately 16-20 h ( equilibration time) after implantation of the probes, microdialysis studies were conducted on freely moving mice.

To reduce exogenous hydroxyl radical formation, the tips of the Hamilton syringes were replaced by polyetheretherketone (PEEK) tips and PEEK tubings (MAB Microbiotech, Stockholm, Sweden). The perfusion fluid was always freshly prepared. Samples were stored at $-20^{\circ} \mathrm{C}$ until further analysis.

\subsubsection{In vivo microdialysis experiments}

Samples $(40 \mu \mathrm{l})$ were collected every $20 \mathrm{~min}$ at a flow rate of $2 \mu \mathrm{l} / \mathrm{min}$ into vials containing $10 \mu \mathrm{l}$ antioxidant solution $(3.3 \mathrm{mM}$ L-cystein, $0.3 \mathrm{mM} \mathrm{Na}_{2}$-EDTA and $0.1 \mathrm{M}$ acetic acid). Before the start of the experiment, Ringer's solution was perfused during $40 \mathrm{~min}$ in order to exclude contamination of the microdialysis system. As 2,3-DHBA is not endogenously present in the brain, no 2,3-DHBA was detected in the dialysates. Afterwards, the perfusion fluid was switched to sodium salicylate ( $1 \mathrm{mM}$, dissolved in Ringer's solution) and 5 dialysate samples were collected before any pharmacological manipulation was performed. The mean of these 2,3-DHBA dialysate concentrations was taken as baseline value.

For the determination of radical scavenging properties, after collecting the baseline samples, 6-OHDA (dissolved in Ringer's solution containing salicylate) was perfused for three collection periods $(1 \mathrm{~h})$ after which the perfusion fluid was switched back to one containing salicylate until the end of the experiment. Two different 6-OHDA concentrations were tested ( $5 \mu \mathrm{M}$ and $50 \mu \mathrm{M})$.

The radical scavenging properties of GSH $(1.5 \mathrm{mM})$ (Soto-Otero et al., 2000) were tested in this model by co-perfusion with sodium salicylate throughout the experiment.

For the determination of radical formation after acute 6-OHDA induced oxidative stress, baseline samples were first collected during salicylate perfusion. Then, Ringer's solution was perfused during $1 \mathrm{~h}$ to wash out salicylate ions. After this wash-out period, 6-OHDA ( $1 \mathrm{mM}$, dissolved in Ringer's solution) was perfused for 15 min, after which the perfusion fluid was switched back to one containing salicylate until the end of the experiment (control group). The choice of $1 \mathrm{mM}$ 6-OHDA for reverse-microdialysis is based on the following reasoning. When $1 \mathrm{mM} 6-\mathrm{OHDA}$ is perfused for $20 \mathrm{~min}$ at $2 \mu \mathrm{l} / \mathrm{min}$, a total dose of about $5 \mu \mathrm{g} / \mathrm{mouse}$ is administered (when considering a relative recovery of $100 \%$ ). The relative recovery of 6-OHDA was estimated from literature findings of the relative recovery of DA for a similar microdialysis set-up. The rationale for this was that 6-OHDA differs from DA only by an hydroxyl group, and therefore should have similar recovery val- ues. Based on this logic, we estimated that the relative recovery of 6-OHDA during our microdialysis experiments was about $20 \%$ (Goldberg et al., 2003), leading to an actual administered amount by reverse-microdialysis of $1 \mu \mathrm{g} /$ mouse.

To validate this method, GSH $(1.5 \mathrm{mM})$ perfusion during the whole experiment was used to test whether the effects of 6-OHDA could be blocked. Alternatively, in another experiment, $\mathrm{CHO}(0.44 \%$ in Ringer's solution) was administered via the microdialysis probe during $40 \mathrm{~min}$ (corresponding to a total CHO dose of $350 \mu \mathrm{g} / \mathrm{mouse}$, similar with the one used by Masukawa et al., 1989) before starting the perfusion with the salicylate in order to investigate whether GSH depletion resulted in enhanced hydroxyl radical formation after 6-OHDA.

\subsubsection{Chromatographic assay}

Determination of 2,3-DHBA concentrations in dialysates was performed using the liquid chromatography (LC) method described by (Yuan et al., 2004), with slight modifications. Twenty $\mu$ l of the sample were injected and analyzed directly for 2,3-DHBA content on a narrowbore (C18 column: $15 \mu \mathrm{m}, 150 \mathrm{~mm} \times 2.1 \mathrm{~mm}$; Altima; Grace; Lokeren; Belgium) LC system. The mobile phase consisted of $0.1 \mathrm{M}$ sodium acetate trihydrate, $20 \mathrm{mM}$ citric acid monohydrate, $1 \mathrm{mM}$ 1-octane sulfonic acid, $0.1 \mathrm{mM} \mathrm{Na}_{2}$ EDTA and $1 \mathrm{mM}$ dibutylamine, adjusted to $\mathrm{pH}$ 3.0. No organic modifier was added. The flow rate was set at $0.3 \mathrm{ml} / \mathrm{min}$. The electrochemical detection (Antec, The Netherlands) potential was $+700 \mathrm{mV}$ versus the reference electrode $(\mathrm{Ag} / \mathrm{AgCl})$. Sensitivity was set at $0.1 \mathrm{nA}$ full scale. All samples were injected via a high precision auto-injector equipped with a cooling system (Kontron, San Diego, CA, USA). The integration of the chromatograms was done with the Data Apex Clarity software program (Antec).

\subsection{Local administration of 6-OHDA by striatal stereotaxic microinjection}

Mice were anesthetized as described above. The skull was exposed and a burr hole was drilled to introduce a $10 \mu$ l calibrated microsyringe (Hamilton CO, Reno, USA) for injection of the 6-OHDA solution (containing $1 \mu \mathrm{g}$ 6-OHDA.HBr per $\mu$ in Ringer's solution). To minimize variability due to degradation of the toxin, the 6-OHDA solutions were freshly prepared, kept on ice, and protected from exposure to light. The solution was injected in the left striatum at the following coordinates relative to the bregma $\mathrm{L}:-1.8, \mathrm{~A}:+0.6$ and $\mathrm{V}:+2.0$, according to the atlas of Franklin and Paxinos (Franklin and Paxinos, 1997). A total volume of $1.5 \mu \mathrm{l}$ 6-OHDA.HBr was injected at a flow rate of $0.5 \mu \mathrm{l} / \mathrm{min}$, leading to a total administered dose of 6 -OHDA of $1 \mu \mathrm{g}$. The syringe was left in place for $5 \mathrm{~min}$ and then slowly removed over a 1-2 min time period. The skin was sutured, and the mice were allowed to recover before returning to the animal housing facilities. Three weeks later, the mice were killed with an overdose of pentobarbital ( $1 \mathrm{ml} \mathrm{Nembutal}{ }^{\circledR}$, Ceva Sante Animale, Brussels, Belgium), and evaluated in terms of the integrity of the nigrostriatal dopaminergic system.

\subsection{Neurochemical determination of the striatal dopamine (DA) and dihydroxyphenylacetic acid (DOPAC) content}

To establish the extent of DA depletion in the mouse striatum after 6-OHDA perfusion, the method described by Yuan et al. (2004) was used.

\subsubsection{Sacrifice and brain prelevation}

The mice from the control group for determining radical formation after acute 6-OHDA induced oxidative stress were kept during 3 weeks after the microdialysis experiment. After 3 weeks, the mice were killed by cervical dislocation and the brains were 
quickly removed without perfusion. From the rostral part of the brain the left and right striatum were dissected out on an ice cold chilled petri-dish, immediately snapfrozen and stored separately at $-80^{\circ} \mathrm{C}$ for homogenisation.

\subsubsection{Homogenisation and HPLC analysis}

DA and DOPAC content were determined in the striatal homogenates. The tissue was wet weighed and a volume of $380 \mu \mathrm{l}$ antioxidant $\left(0.05 \mathrm{M} \mathrm{HCl}, 0.5 \% \mathrm{Na}_{2} \mathrm{~S}_{2} \mathrm{O}_{5}\right.$ and $0.05 \% \mathrm{Na}_{2}$ EDTA) and $20 \mu \mathrm{l}$ internal standard dihydroxybenzylamine (dissolved in antioxidant mixture) $(100 \mathrm{ng} / 100 \mu \mathrm{l})$ was added. The tissue was homogenized and centrifuged at $10,000 \times \mathrm{g}$ for $20 \mathrm{~min}$ at $4{ }^{\circ} \mathrm{C}$. The supernatants were diluted $(1 / 5)$ with acetic acid $(0.5 \mathrm{M})$ and $20 \mu \mathrm{l}$ of sample were injected and analyzed directly for DA and DOPAC content on a similar HPLC system as described in Section 2.2.3. The only difference is that the buffer of the mobile phase was adjusted to $\mathrm{pH} 3.7$ and that methanol (3\%) was used as organic modifier.

The tissue DA and DOPAC content were calculated and expressed as $\mu \mathrm{g} / \mathrm{g}$ wet weight of tissue.

\subsection{Visualization of striatal tyrosine hydroxylase $(\mathrm{TH})$ immunoreactive (ir) nerve terminals}

Three weeks after lesioning, mice were killed with an overdose of pentobarbital ( $1 \mathrm{ml} \mathrm{Nembutal}{ }^{\circledR}$, Ceva Sante Animale, Brussels, Belgium), and perfused transcardially with saline for $5 \mathrm{~min}$, followed by $4 \%$ formaldehyde for $10 \mathrm{~min}$. The formaldehyde solution was prepared from paraformaldehyde on the day of the experiment. During perfusion, $0.1 \mathrm{ml}$ heparin 5000 I.U./ml (LEO Pharma, Belgium) was injected in the spleen of the mice. The brains were removed, placed in vials containing $4 \%$ formaldehyde for 3 days and then cut using a vibratome (Leica Microsystems, Germany), in order to obtain $50 \mu \mathrm{m}$ coronal sections. The immunohistochemical method used was based on the ABC peroxidase technique. Briefly, selected striatal sections were washed three times, each for $5 \mathrm{~min}$, in Tris-saline (0.01 M Tris- $\mathrm{HCl}, 0.1 \%$ Triton X-100, $\mathrm{pH} 7.4)$, and underwent a permeabilizing treatment consisting of incubation in $0.1 \%$ trypsin (Fluka, Buchs, Switzerland) for $1 \mathrm{~h}$ at $37^{\circ} \mathrm{C}$. The sections were then washed twice, each for $5 \mathrm{~min}$, in Tris-saline, followed by quenching of endogenous peroxidase with $3 \% \mathrm{H}_{2} \mathrm{O}_{2}$ for $30 \mathrm{~min}$. The sections were again washed twice, each for $5 \mathrm{~min}$, in Tris-saline, after which non-specific binding was blocked with normal goat serum (diluted 1:5; Millipore, Temecula, CA, USA) for $45 \mathrm{~min}$. This was followed by incubation with rabbit polyclonal anti-TH antibody (AB152; Chemicon, Temecula, CA, USA) at room temperature overnight. After washing twice, each for $5 \mathrm{~min}$, in Tris-saline, the sections were incubated with the biotinylated secondary antibody (Vectastain rabbit IgG ABC kit) for $30 \mathrm{~min}$, followed by two $5 \mathrm{~min}$ washes in Tris-saline. The horseradish peroxidase conjugate (Vectastain rabbit IgG $A B C$ kit) was applied for $30 \mathrm{~min}$, followed by two rinses in Tris-saline, each for 5 min. After a final 5 min rinsing step with acetate buffer, 3,3'-diaminobenzidine (Sigma-Aldrich, Brussels, Belgium) was applied until staining was optimal as determined by light microscopy. The sections were then washed with Tris-saline, dehydrated through a series of ethanol (5 min each in $70 \%, 98 \%, 100 \%$, and $100 \%$ ethanol), cleared with xylene ( $5 \mathrm{~min}$ in xylene, two times), and coverslipped using DPX mountant for histology (Sigma-Aldrich, Brussels, Belgium). After TH staining, images of the sections were digitized with a camera (Sony DXC, Sony Belgium) connected to the light microscope (Yuan et al., 2004).

\subsection{Data analysis}

For the microdialysis data, the striatal extracellular 2,3-DHBA levels were expressed in nM. No corrections were made for probe recovery across the dialysis membrane. The reported extracellular concentrations are actually dialysate concentrations. For statistical analysis, each group was analyzed with one-way analysis of variance (ANOVA) for repeated measures followed by the Dunns post hoc test for comparing the baseline value at time point zero (dialysate fraction 1) and the values during and after the administration of the neurotoxin.

An unpaired Student's $t$-test was applied to compare the means of 2,3-DHBA during the perfusion with 6-OHDA between the experimental groups.

To compare the striatal DA content and the DOPAC:DA ratio the values for intact and lesioned striatum were compared using a two-tailed Student's $t$-test for paired samples. The degrees of lesion of the different experimental groups were compared using a one-way ANOVA followed by a Dunnett post hoc test comparing the microdialysis sham group with the other groups. All statistical analysis was performed with GraphPad Instat 3.0 (GraphPad Prism Software, Inc., San Diego, USA) at the $5 \%$ level of significance.

\section{Results}

3.1. In vivo salicylate trapping to study the radical scavenging properties of compounds during 6-OHDA perfusion in the mouse striatum (Fig. 2)

The perfusion with salicylate $(1 \mathrm{mM}$, during $2 \mathrm{~h})$ produced stable baseline levels of 2,3-DHBA in all the groups and averaged $16.9 \pm 1.1 \mathrm{nM}[$ mean $\pm \operatorname{SEM}(n=12)]$. No significant differences were observed in baseline values of 2,3-DHBA between the groups. After local perfusion with 6-OHDA $(5 \mu \mathrm{M})$ in the mouse striatum for $1 \mathrm{~h}$, the 2,3-DHBA concentration significantly increased by approximately $70 \%$ above the baseline value. Twenty minutes after switching back to salicylate perfusion, the 2,3-DHBA concentration had returned to the baseline level. On the other hand, perfusion with the higher concentration of 6-OHDA $(50 \mu \mathrm{M})$ produced a 7 fold increase in 2,3-DHBA concentrations (data not shown).

Perfusion with the radical scavenger GSH $(1.5 \mathrm{mM})$ did not affect the basal levels of 2,3-DHBA but significantly prevented the increase in the 2,3-DHBA dialysate concentrations during the perfusion with 6-OHDA. These data show that GSH prevents the 6-OHDA induced hydroxyl radical formation.

\subsection{In vivo salicylate trapping to determine hydroxyl radical formation and susceptibility of dopaminergic nerve terminals after striatal 6-OHDA perfusion (Figs. 3 and 4)}

The perfusion with salicylate $(1 \mathrm{mM}$, during $2 \mathrm{~h}$ ) produced a stable baseline level of 2,3-DHBA [14.0 $\pm 0.4 \mathrm{nM}$ (mean \pm SEM), $n=7$ ]. After local perfusion with 6-OHDA ( $1 \mathrm{mM}$, in Ringer's solution without salicylate) in the striatum of the freely moving mice for $15 \mathrm{~min}$, the 2,3-DHBA concentration in the first collected sample under salicylate significantly increased by approximately 5 -fold and remained significantly elevated for an additional $40 \mathrm{~min}$ before returning back to baseline values.

Intrastriatal perfusion of 6-OHDA ( $1 \mathrm{mM})$ significantly reduced striatal DA and DOPAC levels by $30.0 \pm 8.5 \%$ [(mean \pm SEM), $n=6$ ] (Fig. 4A) and 28.3 $\pm 10.3 \%$ [(mean \pm SEM), $n=6$ ] (Fig. 4B), respectively, 3 weeks post-microdialysis when compared to the contralateral intact striatum. No significant differences were observed in DA turnover, which is reflected by the DOPAC:DA ratio, between the intact and denervated side [denervated striatum: $0.055 \pm 0.003$; intact striatum: $0.054 \pm 0.001$, mean $\pm \operatorname{SEM}(n=6)]$.

In order to determine the effect of the stereotaxic surgery and the implantation of the microdialysis probe on the long-term DA and DOPAC levels, we also included a sham group where we perfused Ringer's solution instead of 6-OHDA. Intrastriatal perfusion 


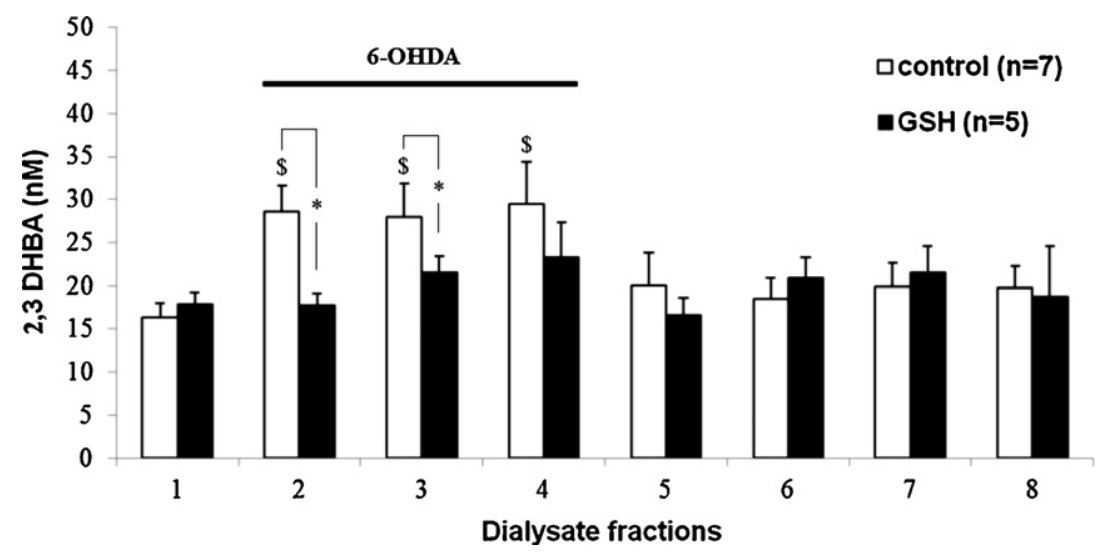

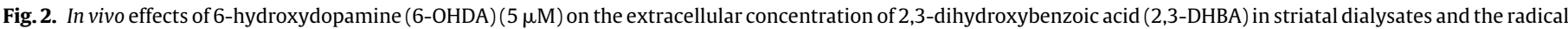

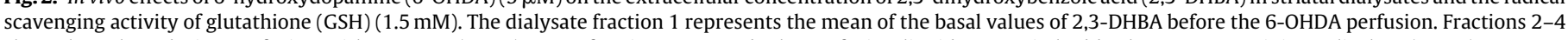

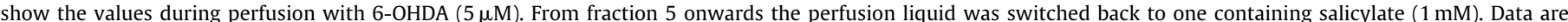

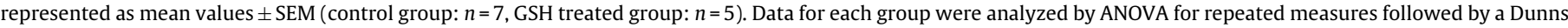

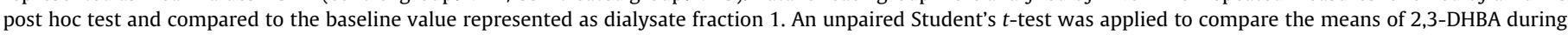

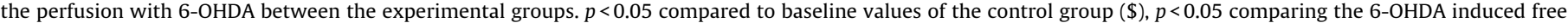
hydroxyl radical formation in control and GSH treated group $\left(^{*}\right)$.
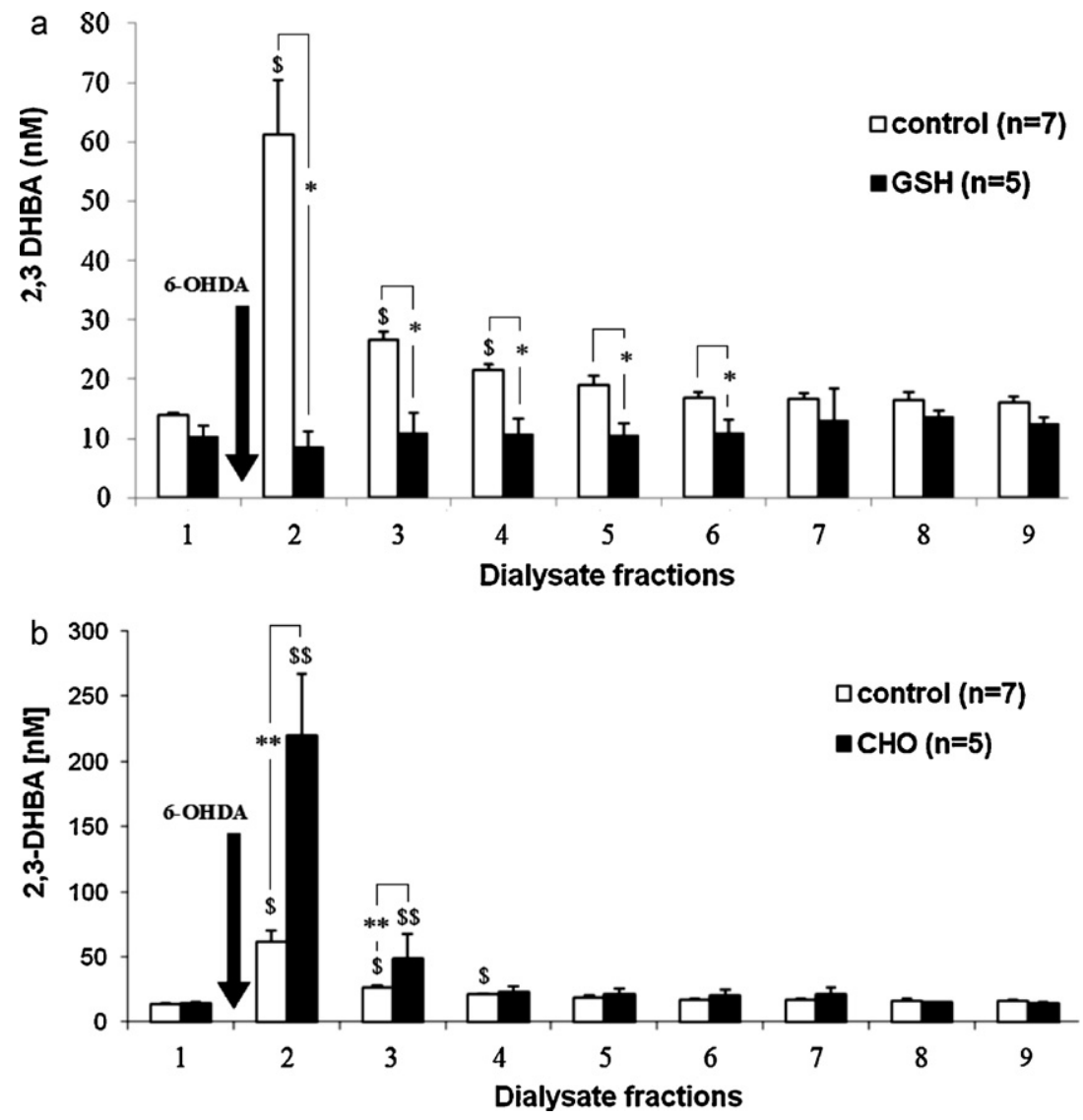

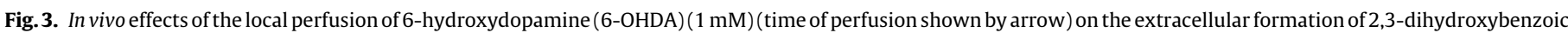

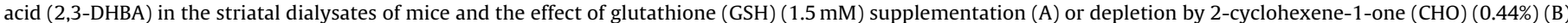

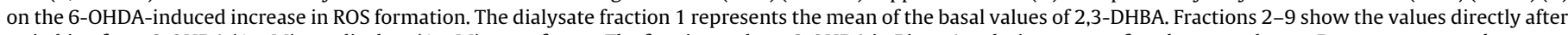

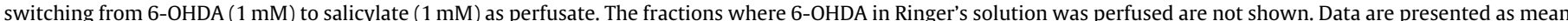

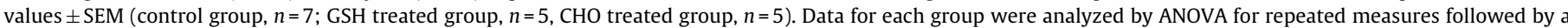

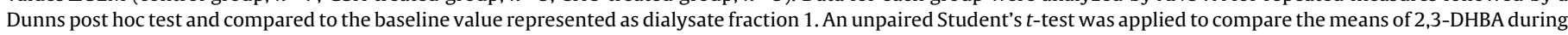

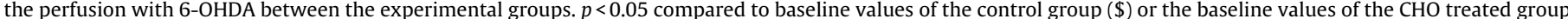

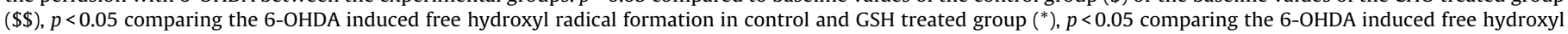
radical formation in control and $\mathrm{CHO}$ treated group $\left({ }^{* *}\right)$. 

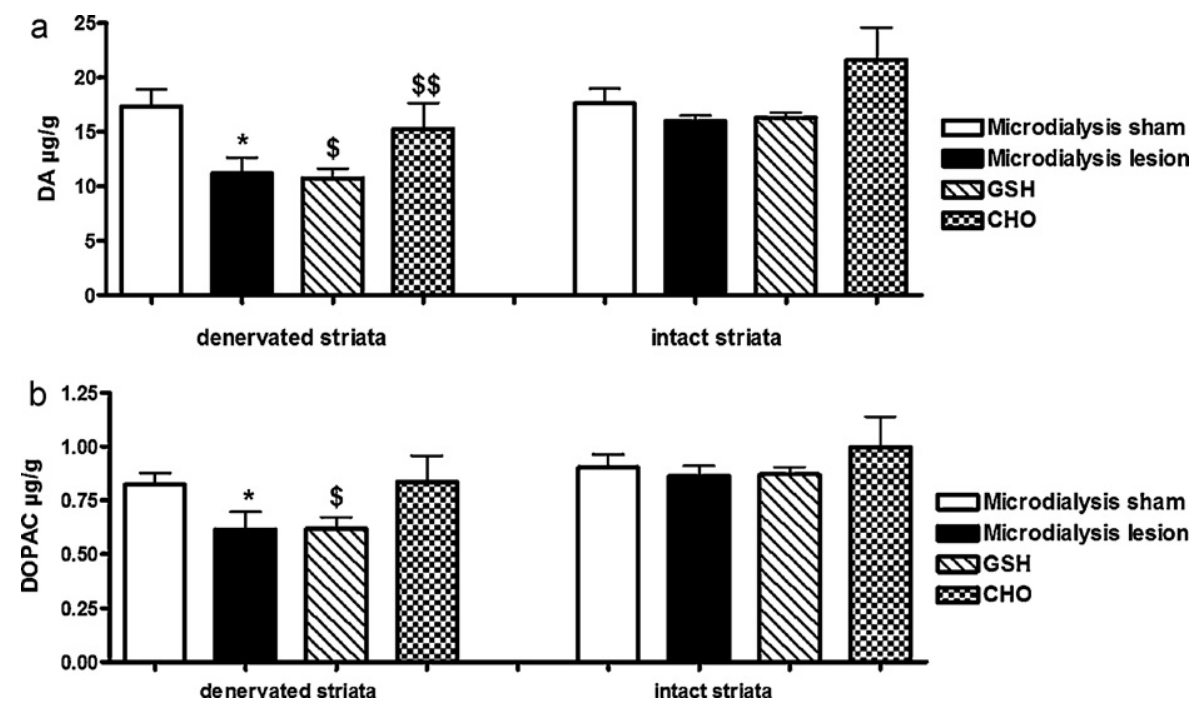

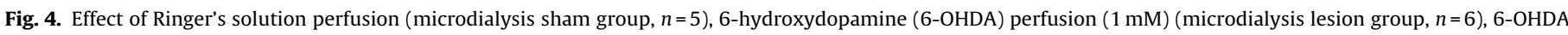

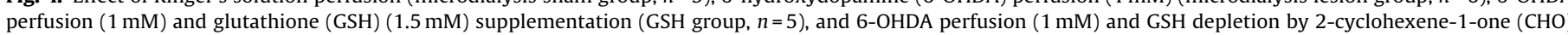

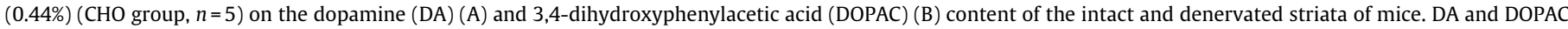

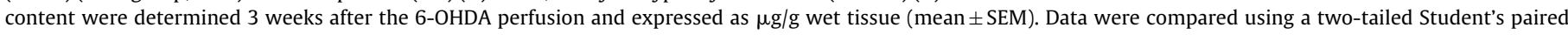

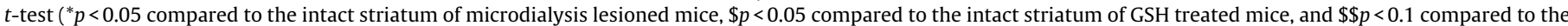
intact striatum of $\mathrm{CHO}$ treated mice).

of Ringer's solution did not significantly reduce the striatal DA and DOPAC levels 3 weeks post-microdialysis when compared to the contralateral intact striatum (Fig. 4A and B). No significant differences were observed in DA turnover between the intact and denervated side [denervated striatum: $0.050 \pm 0.008$; intact striatum: $0.052 \pm 0.011$, mean $\pm \operatorname{SEM}(n=5)]$.

Furthermore, the DA and DOPAC levels of the intact striatum of the sham group were not significantly different from those of the group lesioned by reverse-microdialysis (data not shown). In addition, the degree of lesion in the mice lesioned by reverse-microdialysis of 6-OHDA was significantly higher than that observed in the microdialysis sham group (Table 1 ).

Perfusion with the radical scavenger GSH did not affect the basal levels of 2,3-DHBA before 6-OHDA perfusion, but significantly prevented the 6-OHDA induced hydroxyl radical formation as reflected by the unaltered 2,3-DHBA concentration of the first dialysate sample after the 6-OHDA administration. The values after 6-OHDA perfusion were significantly different from the control group for an additional $80 \mathrm{~min}$, as shown in Fig. 3A.

Intrastriatal 6-OHDA perfusion $(1 \mathrm{mM})$ and $\mathrm{GSH}(1.5 \mathrm{mM})$ supplementation significantly reduced striatal DA and DOPAC levels by $21.7 \pm 13.1 \%$ [(mean \pm SEM), $n=5]$ (Fig. 4A) and $28.6 \pm 5 \%$ [(mean \pm SEM), $n=5$ ] (Fig. 4B), respectively, 3 weeks post-microdialysis when compared to the contralateral intact striatum. No significant differences were observed in DA turnover, between the intact and denervated side [denervated striatum: $0.058 \pm 0.005$; intact striatum: $0.053 \pm 0.002$, mean $\pm \operatorname{SEM}(n=5)]$.

Table 1

Overview of the degrees of lesion in the experimental groups. Degree of dopamine (DA) depletion in the denervated striatum of mice was calculated as a percentage of the DA content in the intact striatum. Data are expressed as mean \pm SEM. Data were compared using one-way ANOVA followed by a Dunnett post hoc test.

\begin{tabular}{lc}
\hline Group & Degree of lesion (\%) \\
\hline Microdialysis sham $(n=5)$ & $0.7 \pm 6.5$ \\
Microdialysis lesion $(n=6)$ & $35.4 \pm 8.2^{\mathrm{a}}$ \\
GSH $(n=5)$ & $34.0 \pm 5.6^{\mathrm{a}}$ \\
CHO $(n=5)$ & $27.5 \pm 7.8^{\mathrm{a}}$ \\
Classical lesion $(n=4)$ & $29.5 \pm 9.2^{\mathrm{a}}$ \\
\hline
\end{tabular}

a $p<0.05$, compared to microdialysis sham mice.
The positive effects of GSH $(1.5 \mathrm{mM})$ supplementation on the acute intrastriatal perfusion of 6-OHDA $(1 \mathrm{mM})$ as observed during salicylate trapping microdialysis were not maintained on longterm, as the degree of lesion of the GSH supplemented mice was not significantly different from untreated mice (microdialysis lesion) (Table 1).

CHO treatment had no effect on the baseline values of 2,3-DHBA. However, after local perfusion with 6-OHDA ( $1 \mathrm{mM}$ ) during $15 \mathrm{~min}$ into the striatum of mice, the 2,3-DHBA concentration significantly increased above baseline values by 16 fold and took longer to reach baseline values when compared to the control group, as shown in Fig. 3B.

Intrastriatal 6-OHDA perfusion ( $1 \mathrm{mM})$ and GSH depletion by $\mathrm{CHO}(0.44 \%)$ reduced striatal DA and DOPAC levels by $27.5 \pm 10.1 \%$ [(mean \pm SEM), $n=5$ ] (Fig. 4A) and $11.4 \pm 14.5 \%$ [(mean \pm SEM), $n=5$ ] (Fig. 4B), respectively, although not to a significant degree ( $p$ value for $\mathrm{DA}=0.1216$ ). No significant differences were observed in DA turnover, between the intact and denervated side [denervated striatum: $0.056 \pm 0.006$; intact striatum: $0.046 \pm 0.003$, mean $\pm \operatorname{SEM}(n=5)]$.

The negative effects of GSH depletion on the acute intrastriatal perfusion of 6-OHDA ( $1 \mathrm{mM})$ as observed during salicylate trapping microdialysis were not maintained on long-term, as the degree of lesion of the GSH depleted mice was not significantly different from untreated mice (microdialysis lesion) (Table 1 ).

\subsection{Effect of striatal micro-injection of 6-OHDA on the DA and DOPAC content of the intact and denervated striatum of mice (Fig. 5)}

In order to validate the lesioning by reverse-microdialysis, we performed a classical 6-OHDA lesion by stereotaxically injecting the same amount of the neurotoxin in the striatum, as that administered during in vivo salicylate trapping microdialysis.

Intrastriatal micro-injection of 6-OHDA ( $1 \mu \mathrm{g}$ free base) significantly reduced striatal DA levels by $29.5 \pm 9.2 \%$ [( mean \pm SEM $)$, $n=4]$ and reduced DOPAC levels by $9.9 \pm 7.1 \%$ [ $($ mean \pm SEM $), n=4]$ although not significantly, 3 weeks post-microdialysis when compared to the contralateral intact striatum (Fig. 5). No significant differences were observed in DA turnover, between the intact and 

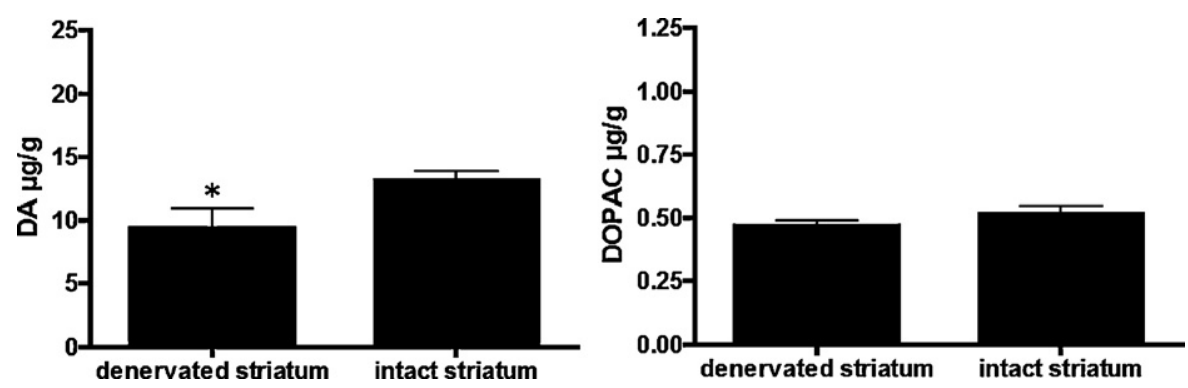

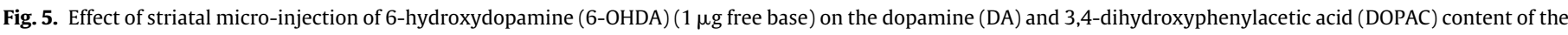

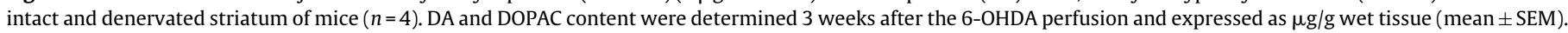
Data were compared using a two-tailed Student's paired $t$-test $\left({ }^{*} p<0.05\right.$ compared to intact striatum).

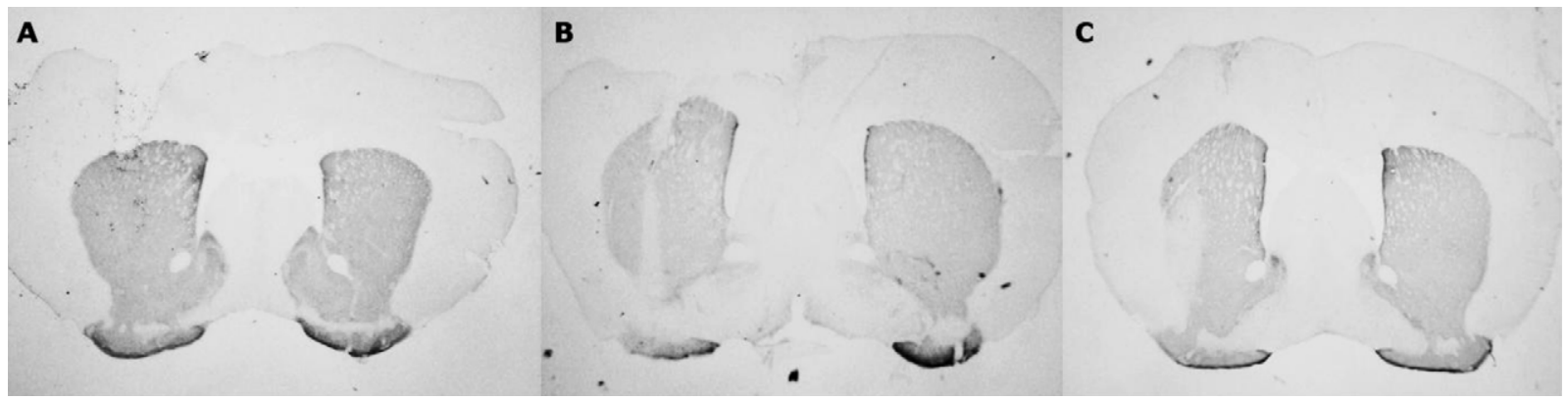

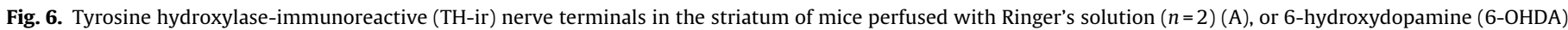

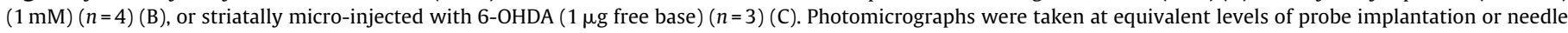
insertion (original magnification $10 \times \times 1.25$ ).

denervated side [denervated striatum: $0.056 \pm 0.010$; intact striatum: $0.040 \pm 0.001$, mean $\pm \operatorname{SEM}(n=4)]$.

The degree of lesion observed in the classically lesioned mice was not significantly different when compared to that of mice lesioned by reverse-microdialysis (Table 1 ).

\subsection{Effect of striatal perfusion with Ringer's solution or 6-OHDA, and of striatal micro-injection of 6-OHDA on TH-ir nerve terminalsin the striatum of mice (Fig. 6)}

Intrastriatal perfusion of Ringer's solution did not affect the THir striatal nerve terminals 3 weeks post-microdialysis (Fig. 6A). On the other hand, intrastriatal perfusion with 6-OHDA $(1 \mathrm{mM})$ led to observable loss of TH staining at the level of the perfusion location (Fig. 6B), which was comparable to that observed after stereotaxic micro-injection with 6-OHDA ( $1 \mu$ g free base) (Fig. $6 \mathrm{C})$. The loss of $\mathrm{TH}$ staining was slightly more diffuse in the classically lesioned mice, and more localized around the perfusion area in the microdialysis lesioned mice.

\section{Discussion}

Hydroxyl radicals are the most reactive and damaging free radicals and may be part of Parkinson's disease pathogenesis (Teismann and Ferger, 2000). In the present study, the in vivo salicylate trapping microdialysis technique was miniaturized for two different applications in mice. First, a method was validated to detect the radical scavenging properties of compounds on hydroxyl radical formation provoked by 6-OHDA perfusion in the mouse striatum. Second, the method was modified so it could be used to determine 6-OHDA induced hydroxyl radical formation in the striatum and the sensitivity of the dopaminergic nerve terminals to this acute oxidative stress. By avoiding simultaneous administration of 6-OHDA together with the trapping agent, salicylate, the neurotoxin was able to maximally challenge the neuronal microenvironment. This allows for an unbiased study on the primary effects of acute 6OHDA induced oxidative stress mediated by the hydroxyl radical formation. This second method can be considered as a new application of the in vivo salicylate trapping microdialysis, as it also allows the investigation of the long-term effects of 6-OHDA administered through the microdialysis probe.

Cheng et al. (2002) reviewed all the methods that are used for the detection of in vivo hydroxyl radical generation. Radical scavenging activity in mice has already been applied in several studies using an intraperitoneal (i.p.) administration of 1-methyl4-phenyl-1,2,3,6-tetrahydropyridine (MPTP) (Ferger et al., 1999; Lu et al., 2008; Thomas et al., 2000). Generally, the in vivo hydroxyl radical generation in the striatum of mice was analyzed by administrating salicylate i.p. just before sacrificing the mice or performing microdialysis and determining in real-time the hydroxyl radical generation by striatal perfusion with salicylate (Ferger et al., 1999; Lu et al., 2008; Thomas et al., 2000). In another study, the systemic administration of 3,4-methylendioxymetamphetamine induced free radical formation in mouse striatum was studied with the in vivo salicylate trapping microdialysis (Camarero et al., 2002).

The choice for salicylate as trapping agent was motivated by the fact that we specifically wanted to capture the hydroxyl radicals. Four-hydroxybenzoic acid for instance also captures other reactive oxygen species, e.g. peroxynitrite. Furthermore, the salicylate trapping microdialysis technique is the most widely used technique for determining hydroxyl radicals in vivo, and it has previously been employed numerous time in animal models of PD (Di Giovanni et al., 2009).

No study was found using 6-OHDA in combination with the in vivo salicylate trapping microdialysis in the mouse striatum. Nevertheless, the use of 6-OHDA in this set-up is of general interest as it is becoming the preferred neurotoxin to create mice models of Parkinson's disease compared to the widely used MPTP. Several reasons to avoid the use of MPTP are proposed, including its strain-, age- and gender-dependency in mice (Alvarez-Fischer et al., 
2008; Grealish et al., 2010; Iancu et al., 2005). For this reason, the in vivo salicylate trapping technique was adapted to investigate compounds for their scavenging activity on 6-OHDA-induced ROS formation in the mouse striatum. Previously, we used $50 \mu \mathrm{M}$ 6-OHDA (Yuan et al., 2004) to induce hydroxyl radical formation in rats. However, in mice, $50 \mu \mathrm{M}$ caused extremely high increases in 2,3-DHBA levels in the dialysates. This was accompanied by the appearance of interfering peaks and the reduced resolution of the 2,3-DHBA peak in the LC chromatograms, hampering its correct determination. Hydroxyl radical formation with $5 \mu \mathrm{M} 6$-OHDA increased by about $70 \%$ from baseline and was therefore chosen in the experiments assaying possible radical scavenging properties of compounds. As expected (Soto-Otero et al., 2000), GSH administration $(1.5 \mathrm{mM})$ was able to prevent hydroxyl radical formation induced by 6-OHDA.

Secondly, we wanted to use the method to determine the effect of 6-OHDA itself on hydroxyl radical formation in the mouse striatum, However, an important disadvantage of the radical scavenging method is that the administered amount of 6-OHDA is too small to induce a significant striatal lesion. Indeed, the delivered amount of 6-OHDA is approximately $3.2 \mathrm{pg}$ within $1 \mathrm{~h}$, while for the striatal lesioning of mice up to $6 \mu \mathrm{g}$ in total are usually administered locally within 5-6 min (Alvarez-Fischer et al., 2008).

Furthermore, the use of a trapping agent such as sodium salicylate may reduce the capacity of the neurotoxin to induce neurodegeneration in the striatum of the mice. Therefore, we administered the same amount of 6-OHDA as the one used for striatal lesioning of mice, in the absence of salicylate. This approach allowed to make a correlation between the acute effects of 6-OHDA and its long-term effects previously described (Alvarez-Fischer et al., 2008; Grealish et al., 2010; Iancu et al., 2005), such as nigral degeneration and reduced striatal DA content.

Indeed, a reproducible 5-fold increase in 2,3-DHBA levels was observed after perfusion with 6-OHDA. This was associated with a significant reduction of approximately $30 \%$ in both DA and DOPAC content in the striatum of these mice, 3 weeks post-microdialysis, while no changes were observed in DA turnover. Importantly, stereotaxic surgery and the implantation of the microdialysis probe had no effect on these parameters, as no lesion was observed in the microdialysis sham group after 3 weeks.

The administration of GSH prevented the 6-OHDA induced oxidative stress and the depletion of GSH after $\mathrm{CHO}$ perfusion rendered the mice more susceptible to oxidative stress. These data confirm GSH to be an important mediator in the prevention of the primary, acute effects of the neurotoxin. This approach can therefore detect differences in the degree of hydroxyl radical formation within the striatum of mice.

Our results show that GSH depletion or supplementation has no effect on the basal production of hydroxyl radicals before 6OHDA stimulation. However, GSH depletion leads to an enhanced 6-OHDA induced hydroxyl radical production that returns slower to basal levels, while GSH supplementation is able to prevent the 6OHDA induced hydroxyl radical production. However, modulation of endogenous GSH levels did not influence the long-term susceptibility to the toxic effects of the neurotoxin. This is in contrast with previous studies, where modulation of GSH levels did affect the long-term effects of 6-OHDA (Garcia et al., 2000; Soto-Otero et al., 2000). Indeed, GSH has previously been shown to protect against neuronal degeneration. In vitro and in vivo data suggest that GSH may protect against DA induced toxicity. GSH treated 6OHDA lesioned rats showed only a decrease of $35 \%$ in dopaminergic terminals 3 weeks after striatal 6-OHDA administration, whereas 6-OHDA alone caused a striatal degeneration of $85 \%$ (Soto-Otero et al., 2000). The neuroprotective actions of GSH are attributed to its central role in the defense against ROS. Since DA metabolism is a source of free radicals, GSH can counter its toxicity by forming conjugates with DA (Jacobsen et al., 2005). GSH may also act as a substrate in the GSH peroxidase mediated destruction of hydroperoxides (Shimizu et al., 2002).

This discrepancy can be due to the fact that during our protocol the GSH levels might have normalized soon after the microdialysis experiment. Therefore, in the described method, the administration of compounds that modulate endogenous antioxidant mechanisms by reverse-microdialysis is not able to change the long-term susceptibility for the toxic effects of 6-OHDA. Nevertheless, the situation is bound to be different when this method is to be applied in genetic animal models of Parkinson's disease. Indeed, as the genetic background of the experimental animals will be maintained during the entire post-lesion period, any observed changes in the acute response to 6-OHDA will be bound to translate into observed changes in the long-term response to the neurotoxin. In line with this hypothesis, our preliminary results obtained from application of the method on PINK-1 knock-out mice, reveal both acute and long-term significant differences when compared to wild-type littermates (unpublished results).

Our results show that lesioning by reverse-microdialysis with $1 \mu \mathrm{g}$ 6-OHDA leads to a similar loss of DA content (30\%) after 3 weeks compared to stereotaxic microinjection of $1 \mu \mathrm{g} 6-\mathrm{OHDA}$ in the striatum. Furthermore, striatal TH staining revealed a similar extent of TH-ir fiber loss following both lesion protocols suggesting reverse-microdialysis can be used as an alternative for the classic lesioning protocol. The advantages of lesioning by reversemicrodialysis are that a single animal can be used to detect both short-term and long-term responses (thereby eliminating interanimal variability) and that it halves the number of experimental animals that have to be used.

No changes were observed in DOPAC:DA ratio for any of our experimental groups. This might be due to the fact that the degree of lesion (which was less than $30 \%$ for all experimental groups) was too low to induce long-term compensatory increases in DA turnover.

Until now, the neurotoxicity of 6-OHDA has been linked to (auto-) oxidation and the formation of quinones, hydrogen peroxide and oxygen radicals, intra- and extracellularly (Blum et al., 2001; Rodriguez-Pallares et al., 2007; Soto-Otero et al., 2000), and to inhibition of complex I and complex IV of the respiratory chain (Themann et al., 2001). Next to this, 6-OHDA has been reported to induce a massive release of DA in the extracellular space of the striatum, which in turn may contribute to hydroxyl radical formation (Ferger et al., 2001). In a recent microdialysis study in mice, enhanced extracellular levels of DA, serotonin and norepinephrine were found after striatal 6-OHDA infusion. An increased level of DA may be correlated with an increased DA metabolism, which in turn may lead to enhance basal production of hydrogen peroxide and cause depleted levels of GSH (Tobon-Velasco et al., 2010). It has also been demonstrated that nigrostriatal infusion of 6-OHDA is associated with an increased inflammatory response, due to activated microglia which produce and release a broad spectrum of free radicals and inflammatory cytokines (Rodriguez-Pallares et al., 2008). The increased oxidative stress within the extracellular space of the mice striatum after 6-OHDA perfusion may be a combination of intra- and extraneuronal derived ROS. Despite all the evidence for a role of oxidative stress in Parkinson's disease, there have been relatively few studies that have extensively characterized oxidative stress in animal models of Parkinson's disease (Smith and Cass, 2007). The currently described methodology might help to distinguish between the several parameters contributing to the hydroxyl radical formation and to further investigate for example the role of microglial activation and the intraneuronal uptake by the DA transporter in oxidative stress. Furthermore, next to toxininduced animal models of Parkinsonism, recent advances in genetic approaches significantly extended the variety of animal models 
by introducing mouse strains carrying specific genetic alterations (Meredith et al., 2008; Sotnikova and Gainetdinov, 2007).

The proposed techniques offer the advantage of a broad application and may contribute to a better characterization of the several available mice models of Parkinson's disease for their susceptibility to oxidative stress. Additionally, it can be of great value as an in vivo tool for screening of possible therapeutic radical scavenging agents. Therefore, this dual approach may be complementary and relevant to other studies.

\section{Conclusion}

In conclusion, the in vivo salicylate trapping microdialysis can be a valuable tool to investigate the importance of oxidative stress as a possible pathogenic factor in Parkinson's disease and other degenerative disorders. The proposed techniques may contribute to a better characterization of the several available mice models of Parkinson's disease, either by providing new opportunities to test potential therapeutic agents, or by characterizing the acute as well as the long-term effects of 6-OHDA in the mouse striatum.

\section{Acknowledgements}

The authors wish to acknowledge the excellent technical assistance of Mrs R. Berckmans, Mr G. De Smet, Mrs C. De Rijck, and Mrs R.-M. Geens. This work was conducted with financial support of the Institute for the promotion of Innovation by Science and Technology in Flanders (IWT) (IWT420), National Fund for Scientific Research (FWO-Vlaanderen) (G.0071.05) and of the Research Council of the Vrije Universiteit Brussel. M. Varçin is holder of a research grant from the IWT (IWT420).

\section{References}

Alvarez-Fischer D, Henze C, Strenzke C, Westrich J, Ferger B, Hoglinger GU, et al. Characterization of the striatal 6-OHDA model of Parkinson's disease in wild type and alpha-synuclein-deleted mice. Exp Neurol 2008;210:182-93.

Aoyama K, Watabe M, Nakaki T. Regulation of neuronal glutathione synthesis. J Pharmacol Sci 2008;108:227-38.

Blum D, Torch S, Lambeng N, Nissou M, Benabid AL, Sadoul R, et al. Molecular pathways involved in the neurotoxicity of 6-OHDA, dopamine and MPTP: contribution to the apoptotic theory in Parkinson's disease. Prog Neurobiol 2001;65:135-72.

Camarero J, Sanchez V, O'Shea E, Green AR, Colado MI. Studies, using in vivo microdialysis, on the effect of the dopamine uptake inhibitor GBR 12909 on 3,4methylenedioxymethamphetamine ('ecstasy')-induced dopamine release and free radical formation in the mouse striatum. J Neurochem 2002;81:961-72.

Cheng FC, Jen JF, Tsai TH. Hydroxyl radical in living systems and its separation methods. J Chromatogr B Analyt Technol Biomed Life Sci 2002;781:481-96.

Choy KH, Dean O, Berk M, Bush AI, van den Buuse M. Effects of N-acetyl-cysteine treatment on glutathione depletion and a short-term spatial memory deficit in 2-cyclohexene-1-one-treated rats. Eur J Pharmacol 2010;649:224-8.

Di Giovanni G, Esposito E, Di Matteo V. In vivo microdialysis in Parkinson's research. J Neural Transm 2009(Suppl.):223-43.

Ferger B, Rose S, Jenner A, Halliwell B, Jenner P. 6-hydroxydopamine increases hydroxyl free radical production and DNA damage in rat striatum. Neuroreport 2001;12:1155-9.

Ferger B, Teismann P, Earl CD, Kuschinsky K, Oertel WH. Salicylate protects against MPTP-induced impairments in dopaminergic neurotransmission at the striatal and nigral level in mice. Naunyn Schmiedebergs Arch Pharmacol 1999;360:256-61.

Ferger B, Teismann P, Mierau J. The dopamine agonist pramipexole scavenges hydroxyl free radicals induced by striatal application of 6-hydroxydopamine in rats: an in vivo microdialysis study. Brain Res 2000;883:216-23.

Franklin K, Paxinos G. The mouse brain in stereotaxic coordinates. San Diego: Academic Press; 1997.

Garcia JC, Remires D, Leiva A, Gonzalez R. Depletion of brain glutathione potentiates the effect of 6-hydroxydopamine in a rat model of Parkinson's disease. J Mol Neurosci 2000;14:147-53.
Goldberg MS, Fleming SM, Palacino JJ, Cepeda C, Lam HA, Bhatnagar A, et al. Parkindeficient mice exhibit nigrostriatal deficits but not loss of dopaminergic neurons. J Biol Chem 2003;278:43628-35.

Grealish S, Mattsson B, Draxler P, Bjorklund A. Characterisation of behavioural and neurodegenerative changes induced by intranigral 6-hydroxydopamine lesions in a mouse model of Parkinson's disease. Eur J Neurosci 2010;31:2266-78.

Gruber M, Wiesner G, Burger R, Lindner R. The salicylate trapping method: is oxidation of salicylic acid solution oxygen and time dependent and metal catalysed? J Chromatogr B Analyt Technol Biomed Life Sci 2006;831:320-3.

Iancu R, Mohapel P, Brundin P, Paul G. Behavioral characterization of a unilateral 6-OHDA-lesion model of Parkinson's disease in mice. Behav Brain Res 2005; $162: 1-10$

Jacobsen JP, Rodriguiz RM, Mork A, Wetsel WC. Monoaminergic dysregulation in glutathione-deficient mice: possible relevance to schizophrenia? Neuroscience 2005; 132:1055-72.

Jenner P. Oxidative stress in Parkinson's disease. Ann Neurol 2003;53(Suppl. 3):S26-36 [Discussion S-8].

Koutsilieri E, Scheller C, Grunblatt E, Nara K, Li J, Riederer P. Free radicals in Parkinson's disease. J Neurol 2002;249(Suppl. 2):II1-5.

Lu KT, Ko MC, Chen BY, Huang JC, Hsieh CW, Lee MC, et al. Neuroprotective effects of resveratrol on MPTP-induced neuron loss mediated by free radical scavenging. J Agric Food Chem 2008;56:6910-3.

Masukawa T, Sai M, Tochino Y. Methods for depleting brain glutathione. Life Sci 1989;44:417-24.

Meredith GE, Sonsalla PK, Chesselet MF. Animal models of Parkinson's disease progression. Acta Neuropathol 2008;115:385-98.

Obata T. Role of hydroxyl radical formation in neurotoxicity as revealed by in vivo free radical trapping. Toxicol Lett 2002;132:83-93.

Obeso JA, Rodriguez-Oroz MC, Goetz CG, Marin C, Kordower JH, Rodriguez M, et al. Missing pieces in the Parkinson's disease puzzle. Nat Med 2010;16:653-61.

Opacka-Juffry J, Wilson AW, Blunt SB. Effects of pergolide treatment on in vivo hydroxyl free radical formation during infusion of 6-hydroxydopamine in rat striatum. Brain Res 1998;810:27-33.

Pileblad E, Magnusson T. Effective depletion of glutathione in rat striatum and substantia nigra by L-buthionine sulfoximine in combination with 2cyclohexene-1-one. Life Sci 1990;47:2333-42.

Przedborski S. Pathogenesis of nigral cell death in Parkinson's disease. Parkinsonism Relat Disord 2005;11(Suppl 1):S3-7.

Rodriguez-Pallares J, Parga JA, Munoz A, Rey P, Guerra MJ, Labandeira-Garcia JL. Mechanism of 6-hydroxydopamine neurotoxicity: the role of NADPH oxidase and microglial activation in 6-hydroxydopamine-induced degeneration of dopaminergic neurons. J Neurochem 2007;103:145-56.

Rodriguez-Pallares J, Rey P, Parga JA, Munoz A, Guerra MJ, Labandeira-Garcia JL. Brain angiotensin enhances dopaminergic cell death via microglial activation and NADPH-derived ROS. Neurobiol Dis 2008;31:58-73.

Schulz JB, Lindenau J, Seyfried J, Dichgans J. Glutathione, oxidative stress and neurodegeneration. Eur J Biochem 2000;267:4904-11.

Shimizu E, Hashimoto K, Komatsu N, Iyo M. Roles of endogenous glutathione levels on 6-hydroxydopamine-induced apoptotic neuronal cell death in human neuroblastoma SK-N-SH cells. Neuropharmacology 2002;43:434-43.

Smith MP, Cass WA. Oxidative stress and dopamine depletion in an intrastriatal 6-hydroxydopamine model of Parkinson's disease. Neuroscience 2007:144:1057-66.

Sotnikova TD, Gainetdinov RR. Microdialysis in genetically altered animals. In: Westerink BHC, TIFHC, editors. Handbook of microdialysis, vol. 16; 2007. p. 399-417.

Soto-Otero R, Mendez-Alvarez E, Hermida-Ameijeiras A, Munoz-Patino AM Labandeira-Garcia JL. Autoxidation and neurotoxicity of 6-hydroxydopamine in the presence of some antioxidants: potential implication in relation to the pathogenesis of Parkinson's disease. J Neurochem 2000;74:1605-12.

Teismann P. Ferger B. The salicylate hydroxylation assay to measure hydroxyl free radicals induced by local application of glutamate in vivo or induced by the Fenton reaction in vitro. Brain Res Brain Res Protoc 2000;5:204-10.

Themann C, Teismann P, Kuschinsky K, Ferger B. Comparison of two independent aromatic hydroxylation assays in combination with intracerebral microdialysis to determine hydroxyl free radicals. J Neurosci Methods 2001;108:57-64.

Thomas B, Muralikrishnan D, Mohanakumar KP. In vivo hydroxyl radical generation in the striatum following systemic administration of 1-methyl-4-phenyl1,2,3,6-tetrahydropyridine in mice. Brain Res 2000;852:221-4.

Tobon-Velasco JC, Silva-Adaya D, Carmona-Aparicio L, Garcia E, Galvan-Arzate S, Santamaria A. Early toxic effect of 6-hydroxydopamine on extracellular concentrations of neurotransmitters in the rat striatum: an in vivo microdialysis study. Neurotoxicology 2010;31:715-23.

Toulouse A, Sullivan AM. Progress in Parkinson's disease-where do we stand? Prog Neurobiol 2008;85:376-92.

Yacoubian TA, Standaert DG. Targets for neuroprotection in Parkinson's disease. Biochim Biophys Acta 2009;1792:676-87.

Yuan H, Sarre S, Ebinger G, Michotte Y. Neuroprotective and neurotrophic effect of apomorphine in the striatal 6-OHDA-lesion rat model of Parkinson's disease. Brain Res 2004;1026:95-107. 